\title{
Erratum
}

\section{A family of polycyclic groups over which the uniform conjugacy problem is NP-complete}

\author{
[Int. J. Algebra Comput., Vol. 24, No. 4 (2014) 515-530, \\ DOI: $10.1142 / \mathrm{S} 0218196714500234]$ \\ Bren Cavallo \\ Department of Mathematics \\ CUNY Graduate Center \\ 365 5th Avenue, New York, 10016, USA \\ bcavallo@gc.cuny.edu \\ Delaram Kahrobaei \\ Department of Computer Science \\ CUNY Graduate Center \\ 365 5th Avenue, New York, 10016, USA \\ Department of Mathematics \\ New York City College of Technology \\ 300 Jay Street, New York, 11201, USA \\ dkahrobaei@gc.cuny.edu
}

Published 17 May 2017

In [1] we attempted to construct a family of polycyclic groups, $\left\{G_{n}\right\}$, such that the uniform conjugacy problem over the $\left\{G_{n}\right\}$ NP-Complete. Unfortunately, it was brought to our attention by the Board that our construction detailed in Section 6 of [1] is flawed in that the maps we use to extend $G_{i}$ to obtain $G_{i+1}$ are not automorphisms which in turn implies that the twisted subset problem (described in Section 7 of the same paper) we obtain following conjugation is trivial. For instance, when we extend $G_{1}$ to $G_{2}$ we have the following problem which we describe in the notation given in Section 6 of [1].

Consider the following words given by the generators of $G_{2}$ :

$$
g_{4} g_{2}\left(g_{1} g_{3}\right) g_{2}^{-1} g_{4}^{-1}=g_{2} g_{4}\left(g_{1} g_{3}\right) g_{4}^{-1} g_{2}^{-1}
$$

which are equivalent as elements of $G_{2}$ since $g_{2}$ and $g_{4}$ commute. Further applying the relations of $G_{2}$ we then have: 


$$
\begin{aligned}
& g_{4} g_{2}\left(g_{1} g_{3}\right) g_{2}^{-1} g_{4}^{-1}= \\
& g_{4} g_{1}^{-1}\left(g_{2} g_{3} g_{2}^{-1}\right) g_{4}^{-1}= \\
& g_{4} g_{1}^{-1}\left(g_{1}^{-1} g_{3}\right) g_{4}^{-1}= \\
& g_{1}^{2} g_{3}
\end{aligned}
$$

and

$$
\begin{aligned}
& g_{2} g_{4}\left(g_{1} g_{3}\right) g_{4}^{-1} g_{2}^{-1}= \\
& g_{2}\left(g_{1}^{-1} g_{3}\right) g_{2}^{-1}= \\
& g_{1}\left(g_{2} g_{3} g_{2}^{-1}\right)= \\
& g_{1}\left(g_{1}^{-1} g_{3}\right)=g_{3}
\end{aligned}
$$

which in turn implies that $g_{1}^{2}=1$ since the original words were equivalent as elements of $G_{2}$. Since $g_{1}^{2}=1$ the twisted subset sum problem we obtain above $g_{1}$ following conjugation (as seen in Theorem 6.2 from [1]) is then trivial. Hence the paper's main result, Theorem 8.4, is incorrect.

To the best of our knowledge, the remaining content of the paper is valid including the results in Section 8 that show that the twisted subset sum problem is NP-Complete.

The authors would like to thank the Editorial Board for bringing our attention to the fact that the construction in Section 6 of [1] is incorrect and does not imply the paper's main result.

\section{Reference}

[1] B. Cavallo and D. Kahrobaei, A family of polycyclic groups over which the uniform conjugacy problem is NP-complete, Int. J. Algebra Comput. 24(4) (2014) 515-530. 